\title{
Distributed vs. Centralized Power Systems Frequency Control
}

\author{
Martin Andreasson $^{12}$, Dimos V. Dimarogonas ${ }^{1}$, Karl H. Johansson ${ }^{1}$ and Henrik Sandberg ${ }^{1}$ \\ *ACCESS Linnaeus Center, KTH Royal Institute of Technology, Stockholm, Sweden.
}

\begin{abstract}
This paper considers a distributed control algorithm for frequency control of electrical power systems. We propose a distributed controller which retains the reference frequency of the buses under unknown load changes, while asymptotically minimizing a quadratic cost of power generation. For comparison, we also propose a centralized controller which also retains the reference frequency while minimizing the same cost of power generation. We derive sufficient stability criteria for the parameters of both controllers. The controllers are evaluated by simulation on the IEEE 30 bus test network, where their performance is compared.
\end{abstract}

\section{INTRODUCTION}

Distributed control is in many large-scale systems the only feasible control strategy, when sensing and actuation communications are limited. One important class of largescale systems are electrical power systems, which employ automatic generation control (AGC) [1], [2] and frequency controllers [3], [4]. The frequency controllers are mainly centralized [5], [3], however some efforts towards distributed control of power system frequencies have been made [6]. Due to load and generation changes as well as model imperfections, a proportional frequency controller cannot reach the desired reference frequency in general. To attenuate static errors, integrators are used [4]. Due to the inherent difficulties with distributed PI control [7], automatic frequency control of power systems is typically carried out at two levels: an inner and an outer level. In the inner control loop, the frequency is controlled with a proportional controller against a dynamic reference frequency. In the outer loop, the reference frequency is controlled with a centralized PI controller to eliminate static errors. While this control architecture works satisfactorily in most of today's situations, future power system developments might render it unsuitable. For instance, large-scale penetration of renewable power generation increases generation fluctuations, creating a need for fast as well as local disturbance attenuation. Distributed control of power systems might also provide efficient anti-islanding control and self-healing features, even when communication between subsystems is limited or even unavailable [8], [9].

This work was supported in part by the European Commission by the Hycon2 project, the Swedish Research Council (VR) and the Knut and Alice Wallenberg Foundation. We would like to thank the anonymous reviewers for their valuable comments. The $2^{\text {nd }}$ author is also affiliated with the Centre for Autonomous Systems at KTH and is supported by the VR 2009-3948 grant. ${ }^{2}$ Corresponding author. E-mail: mandreas@kth.se
In this paper we propose a novel distributed frequency controller for electrical power systems. We extend the distributed frequency controller proposed in [10] to also minimize a quadratic generation cost function, besides regulating the bus frequencies towards a reference frequency under unknown load changes. We derive sufficient conditions based on the eigenvalues of the system matrix under which the power system is asymptotically stable. We also provide sufficient conditions on the parameters of the controller, under which the distributed controller stabilizes the power system. These conditions take the form of scalar inequalities, which are easily verified. We also propose a centralized frequency controller for electrical power systems, and provide sufficient stability conditions for this controller.

The rest of the paper is organized as follows. In section II we introduce the mathematical notation used throughout the paper. In section III we introduce the model and define the problem treated in this work. In section IV we introduce a distributed controller and analyze the stability of the closedloop system. In section V we specify a centralized controller, and provide stability analysis. In section VI we compare the performance of the distributed and centralized controllers by simulations on the IEEE 30 bus test system. The paper ends by some concluding remarks in section VII.

\section{NOTATION}

Let $\mathcal{G}$ be a graph. Denote by $\mathcal{V}=\{1, \ldots, n\}$ the vertex set of $\mathcal{G}$, and by $\mathcal{E}=\{1, \ldots, m\}$ the edge set of $\mathcal{G}$. Let $\mathcal{N}_{i}$ be the set of neighboring vertices to $i \in \mathcal{V}$. Denote by $\mathcal{B}=$ $\mathcal{B}(\mathcal{G})$ the vertex-edge adjacency matrix of $\mathcal{G}$, and let $\mathcal{L}$ be the Laplacian matrix of $\mathcal{G}$. In this paper we will only consider static, undirected and connected graphs. For the application of frequency control of power systems, this is a reasonable assumption as long as there are no power line failures. For undirected graphs it holds that $\mathcal{L}=\mathcal{B B}^{T}$. Let $\mathbb{C}^{-}$denote the open left half complex plane, and $\overline{\mathbb{C}}^{-}$its closure. We denote by $c_{n \times m}$ a vector or matrix of dimension $n \times m$ whose elements are all equal to $c . I_{n}$ denotes the identity matrix of dimension $n$.

\section{MODEL AND PROBLEM SETUP}

We consider a power system consisting of $n$ buses. The topology of the power system is given by the graph $\mathcal{G}=$ $(\mathcal{V}, \mathcal{E})$, where $\mathcal{V}$ is the set of buses and $\mathcal{E}$ is the set of power transmission lines. Let $\delta_{i}$ be the phase angle of bus $i$. The 
dynamics of the power system are assumed to be given by the swing equation [4]:

$$
m_{i} \ddot{\delta}_{i}+d_{i} \dot{\delta}_{i}=-\sum_{j \in \mathcal{N}_{i}} k_{i j} \sin \left(\delta_{i}-\delta_{j}\right)+p_{i}^{m}+u_{i},
$$

where $m_{i}>0$ and $d_{i}>0$ are the inertia and damping coefficient, respectively, of bus $i, p_{i}^{m}$ is the power load and $u_{i}$ is the power generation, $k_{i j}=\left|V_{i}\right|\left|V_{j}\right| b_{i j}$, where $V_{i}$ is the voltage of bus $i$, and $b_{i j}$ is the susceptance of the power line $(i, j)$. By linearizing (1) around the equilibrium where $x_{i}=x_{j} \forall i, j \in \mathcal{V}$, the linearized swing equation is obtained:

$$
m_{i} \ddot{\delta}_{i}+d_{i} \dot{\delta}_{i}=-\sum_{j \in \mathcal{N}_{i}} k_{i j}\left(\delta_{i}-\delta_{j}\right)+p_{i}^{m}+u_{i} .
$$

By defining the state vector $\delta=\left[\delta_{i}, \ldots, \delta_{n}\right]$, and the bus frequencies $\dot{\delta}=\omega$, we may rewrite (2) in state-space form as

$$
\left[\begin{array}{c}
\dot{\delta} \\
\dot{\omega}
\end{array}\right]=\left[\begin{array}{cc}
0_{n \times n} & I_{n} \\
-M \mathcal{L}_{k} & -M D
\end{array}\right]\left[\begin{array}{c}
\delta \\
\omega
\end{array}\right]+\left[\begin{array}{c}
0_{n \times 1} \\
M P^{m}
\end{array}\right]+\left[\begin{array}{c}
0_{n \times 1} \\
M u
\end{array}\right],
$$

where $M=\operatorname{diag}\left(\frac{1}{m_{1}}, \ldots, \frac{1}{m_{n}}\right), D=\operatorname{diag}\left(d_{1}, \ldots, d_{n}\right), \mathcal{L}_{k}$ is the weighted Laplacian with edge weights $k_{i j}, P^{m}=$ $\left[p_{1}^{m}, \ldots, p_{n}^{m}\right]^{T}, u=\left[u_{i}, \ldots, u_{n}\right]^{T}$. Assume that there is a cost $f_{i}^{c}\left(u_{i}\right)=\frac{1}{2} C_{i} u_{i}^{2}$ of generating the power $u_{i}$ at bus $i$. The objective is to design a distributed control protocol that satisfies the following conditions:

Condition 1: The controller asymptotically regulates the bus frequencies to the reference frequency $\omega^{\text {ref }}$, i.e.,

$$
\lim _{t \rightarrow \infty} \omega_{i}(t)=\omega^{\mathrm{ref}} \forall i \in \mathcal{V}
$$

Condition 2: The power generation minimizes the accumulate generation cost in steady state of (3), i.e.,

$$
\lim _{t \rightarrow \infty} u(t)=u^{*}
$$

where $u^{*}$ is the minimizer of

$$
\sum_{i \in \mathcal{V}} \frac{1}{2} C_{i} u_{i}^{2} \quad \text { s.t. } \quad \mathcal{L}_{k} \delta-u=P^{m}-\omega^{\mathrm{ref}} D 1_{n \times 1},
$$

where the constraint assures balance between generated and consumed power in stationarity.

\section{Distributed CONTROL}

\section{A. Proposed control protocol}

We propose the following control protocol:

$$
\begin{aligned}
u_{i}= & \alpha\left(\hat{\omega}_{i}-\omega_{i}\right) \\
\dot{\hat{\omega}}_{i}= & \beta\left(\sum_{j \in \mathcal{N}_{i}} k_{i j} \alpha\left(C_{j}\left(\hat{\omega}_{j}-\omega_{j}\right)-C_{i}\left(\hat{\omega}_{i}-\omega_{i}\right)\right)\right) \\
& +\gamma\left(\omega^{\text {ref }}-\omega_{i}\right)
\end{aligned}
$$

where $\alpha, \beta, \gamma \in \mathbb{R}^{+}$. We will show that the controller (7) satisfies conditions 1 and 2 .

Note 1: The control protocol (7) is distributed, and its communication graph is assumed to be identical with that of the power system.

Note 2: $C_{i} u_{i}$ can be interpreted as the marginal cost of power generation for bus $i$.

\section{B. Sufficient stability criterion based on eigenvalues}

In this section we study the stability of (3) with the control given by (7). We first give sufficient conditions for the stability of the proposed control protocol based on linear system theory.

Theorem 1: The power system (3) with control input (7) satisfies Conditions 1 and 2 for any initial condition $(\delta(0), \omega(0))$ if the matrix

$$
A \triangleq\left[\begin{array}{ccc}
-\alpha \beta \mathcal{L}_{k} C & 0_{n \times n} & \alpha \beta \mathcal{L}_{k} C-\gamma I_{n} \\
0_{n \times n} & 0_{n \times n} & I_{n} \\
\alpha M & -M \mathcal{L}_{k} & -M\left(D+\alpha I_{n}\right)
\end{array}\right]
$$

where $C=\operatorname{diag}\left[c_{1}, \ldots, c_{n}\right]$ has exactly one eigenvalue equal to zero and all other eigenvalues in the open left half complex plane.

Proof: Assume that $A$ has exactly one zero eigenvalue, and all other eigenvalues in the left half complex plane. It can be verified that the dynamics of the system (3) with the control given by (7) can be written as

$$
\left[\begin{array}{c}
\dot{\hat{\omega}} \\
\dot{\delta} \\
\dot{\omega}
\end{array}\right]=A\left[\begin{array}{c}
\hat{\omega} \\
\delta \\
\omega
\end{array}\right]+\left[\begin{array}{c}
\gamma \omega^{\mathrm{ref}} 1_{n \times 1} \\
0_{n \times 1} \\
M P^{m}
\end{array}\right] \text {. }
$$

Consider the linear change of coordinates:

$$
\begin{aligned}
\delta & =\left[\begin{array}{cc}
\frac{1}{\sqrt{n}} 1_{n \times 1} & S
\end{array}\right] \delta^{\prime} \\
\delta^{\prime} & =\left[\begin{array}{c}
\frac{1}{\sqrt{n}} 1_{1 \times n} \\
S^{T}
\end{array}\right] \delta .
\end{aligned}
$$

where $S$ is a matrix such that $\left[\frac{1}{\sqrt{n}} 1_{n \times 1} \quad S\right]$ is an orthonormal matrix. In the new coordinates the system dynamics are given by:

$$
\begin{aligned}
& \dot{\hat{\omega}}=-\alpha \beta \mathcal{L}_{k} C \hat{\omega}+\left(\alpha \beta \mathcal{L}_{k} C-\gamma I_{n}\right) \omega+\gamma 1_{n \times 1} \omega^{\mathrm{ref}} \\
& \dot{\delta}^{\prime}=\left[\begin{array}{c}
\frac{1}{\sqrt{n}} 1_{1 \times n} \\
S^{T}
\end{array}\right] \omega \\
& \dot{\omega}=\alpha M \hat{\omega}-M \mathcal{L}_{k}\left[\frac{1}{\sqrt{n}} 1_{n \times 1} \quad S\right] \delta^{\prime}-M\left(D+\alpha I_{n}\right) \omega+M P .
\end{aligned}
$$

By defining the output of the system (3) and (7) as

$$
y=\left[\begin{array}{c}
\mathcal{L}_{k} \delta \\
\omega
\end{array}\right]=\left[\begin{array}{c}
\left.\mathcal{L}_{k}\left[\begin{array}{c}
\frac{1}{\sqrt{n}} 1_{n \times 1} S \\
\omega
\end{array}\right] \delta^{\prime}\right]=\left[\begin{array}{c}
0 \\
0 \\
\mathcal{L}_{K} s \\
\omega
\end{array}\right] \delta^{\prime} \\
\omega
\end{array}\right.
$$

which are the system states of interest, we note that $\delta_{1}^{\prime}$ is unobservable. Hence we may omit this state by defining $\delta^{\prime \prime}=$ $\left[\delta_{2}^{\prime}, \ldots, \delta_{n}^{\prime}\right]$. In the new coordinates the system dynamics are given by

$$
\left[\begin{array}{c}
\dot{\hat{\omega}} \\
\dot{\delta}^{\prime \prime} \\
\dot{\omega}
\end{array}\right]=A^{\prime}\left[\begin{array}{c}
\hat{\omega} \\
\delta^{\prime \prime} \\
\omega
\end{array}\right]+\underbrace{\left[\begin{array}{c}
\gamma 1_{n \times 1} \\
0_{(n-1) \times 1} \\
M P
\end{array}\right]}_{\triangleq b},
$$

where

$$
A^{\prime}=\left[\begin{array}{ccc}
-\alpha \beta \mathcal{L}_{k} C & 0_{n \times(n-1)} & \alpha \beta \mathcal{L}_{k} C-\gamma I_{n} \\
0_{(n-1) \times n} & 0_{(n-1) \times(n-1)} & S^{T} \\
\alpha M & -M \mathcal{L}_{k} S & -M\left(D+\alpha I_{n}\right)
\end{array}\right] .
$$


We now show that $A$ has full rank. Consider

$$
A\left[\begin{array}{c}
\hat{\omega} \\
\delta^{\prime} \\
\omega
\end{array}\right]=\left[\begin{array}{c}
0_{n \times 1} \\
0_{(n-1) \times 1} \\
0_{n \times 1}
\end{array}\right] .
$$

The second row of the above equation gives $S^{T} \omega=$ $0_{(n-1) \times 1}$, implying $\omega=k 1_{n \times 1}$. The first row gives $\alpha \beta \mathcal{L}_{k} C \hat{\omega}=\left(\alpha \beta \mathcal{L}_{k} C-\gamma I_{n}\right) k 1_{n \times 1}$, which implies $k=0$ since $1_{n \times 1}$ does not lie $\mathrm{i}$ the range of $\mathcal{L}_{k}$. Finally, the third row gives $M \mathcal{L}_{k} S \delta^{\prime}=0_{(n-1) \times 1}$, implying $\delta^{\prime}=0_{(n-1) \times 1}$. Since by the change of coordinates, the eigenvalues of $A$ remain the same, we also conclude that $A^{\prime}$ has the same eigenvalues as $A$, except the zero eigenvalue. It follows that $A^{\prime}$ is Hurwitz iff $A$ has exactly one zero eigenvalue, and all other eigenvalues in the left half complex plane. We now shift the state-space by defining

$$
\left[\begin{array}{c}
\hat{\omega} \\
\delta^{\prime \prime \prime} \\
\omega
\end{array}\right]=\left[\begin{array}{c}
\hat{\omega} \\
\delta^{\prime \prime} \\
\omega
\end{array}\right]-A^{\prime-1} b .
$$

It follows that in these new coordinates, the system dynamics are

$$
\left[\begin{array}{c}
\dot{\hat{\omega}} \\
\dot{\delta}^{\prime \prime \prime} \\
\dot{\omega}
\end{array}\right]=A^{\prime}\left[\begin{array}{c}
\hat{\omega} \\
\delta^{\prime \prime \prime} \\
\omega
\end{array}\right] .
$$

The equilibrium solution of (10) satisfies

$$
\begin{aligned}
\alpha \beta \mathcal{L}_{k} C(\omega-\hat{\omega})-\gamma \omega & =\gamma 1_{n \times 1} \omega^{\text {ref }} \\
S^{T} \omega & =0_{n \times 1} .
\end{aligned}
$$

As the rows of $S^{T}$ are orthonormal to $1_{1 \times n}$, (12) implies that $\omega=c_{1} 1_{n \times 1}$, where $c_{1} \in \mathbb{R}$. Substituting this in (11) yields

$$
\alpha \beta \mathcal{L}_{k} C(\omega-\hat{\omega})-\gamma c_{1} 1_{n \times 1}=\gamma 1_{n \times 1} \omega^{\text {ref }} .
$$

Since $1_{n \times 1}$ is not in the range of $\mathcal{L}_{k}$, we conclude that $c_{1}=$ $\omega^{\text {ref }}$, implying that (4) is satisfied. Furthermore (11) implies $C(\omega-\hat{\omega})=c_{2} 1_{n \times 1}$. The KKT conditions [11] of the convex constrained optimization problem (6) are

$$
C u=C \alpha(\omega-\hat{\omega})=\lambda 1_{n \times 1},
$$

where $\lambda$ is the Lagrange multiplier. Since the equilibrium of (10) implies the KKT conditions, and the KKT conditions are necessary and sufficient optimality conditions, the equilibrium of (8) must be the optimal solution of (6).

\section{Explicit sufficient stability criterion}

While Theorem 1 provides a relatively straightforward condition whether a given set of parameters result in a stable system, it does not suggest how to stabilize an unstable system. In the following section we give sufficient conditions for when $A$ has all eigenvalues except one in the left complex plane.

Theorem 2: $A$ has exactly one zero eigenvalue, and all other eigenvalues in the left half complex plane if the following conditions are satisfied

$$
\begin{aligned}
& \beta \lambda_{\max }\left(\mathcal{L}_{k} C \mathcal{L}_{k}\right) \underline{m} \\
& <\alpha\left(\beta \lambda_{\min }\left(\frac{1}{2}\left(L_{k} C D+D C L_{k}\right)\right)+\gamma\right) . \\
& \left(\beta \lambda_{\min }\left(\frac{1}{2}\left(L_{k} C M^{-1}+M^{-1} C L_{k}\right)\right)+1+\frac{D}{\alpha}\right) \\
& \beta \lambda_{\min }\left(\frac{1}{2}\left(L_{k} C D+D C L_{k}\right)\right)+\gamma>0 \\
& \beta \lambda_{\min }\left(\frac{1}{2}\left(L_{k} C M^{-1}+M^{-1} C L_{k}\right)\right)+1+\frac{D}{\alpha}>0 .
\end{aligned}
$$

where $\underline{m}=\min _{i} m_{i}$ and $\underline{D}=\min _{i} D_{i}$.

Remark 1: Given the power system parameters and the controller gains $\alpha$ and $\gamma$, there always exists $\beta>0$, such that the controller (7) stabilizes the power system.

Proof: The characteristic polynomial of $A$ is given by:

$$
\begin{aligned}
& 0=\left|\begin{array}{ccc}
s I_{n}+\alpha \beta \mathcal{L}_{k} C & 0_{n \times n} & -\alpha \beta \mathcal{L}_{k} C+\gamma I_{n} \\
0_{n \times n} & s I_{n} & -I_{n} \\
-\alpha M & -M \mathcal{L}_{k} & s I_{n}+M\left(D+\alpha I_{n}\right)
\end{array}\right| \\
& =\left|\begin{array}{ccc}
s I_{n}+\alpha \beta \mathcal{L}_{k} C & 0_{n \times n} & (\gamma+s) I_{n} \\
0_{n \times n} & s I_{n} & -I_{n} \\
-\alpha M & -M \mathcal{L}_{k} & \left.s I_{n}+M D\right)
\end{array}\right| \\
& =\frac{1}{s^{n}}\left|\begin{array}{ccc}
s I_{n}+\alpha \beta \mathcal{L}_{k} C & 0_{n \times n} & \left(\gamma s+s^{2}\right) I_{n} \\
0_{n \times n} & s I_{n} & 0_{n \times n} \\
-\alpha M & 0_{n \times n} & \left.s^{2} I_{n}+s M D+M \mathcal{L}_{k}\right)
\end{array}\right| \\
& =\left|\begin{array}{ccc}
s I_{n}+\alpha \beta \mathcal{L}_{k} C & 0_{n \times n} & \left(\gamma s+s^{2}\right) I_{n} \\
0_{n \times n} & I_{n} & 0_{n \times n} \\
-\alpha M-s I_{n}-\alpha \beta \mathcal{L}_{k} C & 0_{n \times n} & \left.s M D-s \gamma I_{n}+M \mathcal{L}_{k}\right)
\end{array}\right| \\
& =\left|\begin{array}{cc}
s I_{n}+\alpha \beta \mathcal{L}_{k} C & \left(\gamma s+s^{2}\right) I_{n} \\
-\alpha M & s^{2} I_{n}+s M D+M \mathcal{L}_{k}
\end{array}\right| \\
& =\operatorname{det}(-\alpha M) \operatorname{det}\left[I_{n}\left(\gamma s+s^{2}\right)\right. \\
& \left.+\left(s I_{n}+\alpha \beta \mathcal{L}_{k} C\right) \frac{1}{\alpha} M^{-1}\left(s^{2} I_{n}+s M D+M \mathcal{L}_{k}\right)\right] \\
& =\operatorname{det}\left(\beta \mathcal{L}_{k} C \mathcal{L}_{k}+s\left(\gamma I_{n}+\frac{1}{\alpha} \mathcal{L}_{k}+\beta \mathcal{L}_{k} C D\right)\right. \\
& \left.s^{2}\left(I_{n}+\frac{1}{\alpha} D+\beta \mathcal{L}_{k} C M^{-1}\right)+s^{3} \frac{1}{\alpha} M^{-1}\right) \triangleq \operatorname{det} Q,
\end{aligned}
$$

where we have used standard properties of determinants [12]. A necessary condition for the above equation to have a solution is that $\exists x: x^{*} Q(s) x=0$. We may without loss of generality assume $x^{*} x=1$. Hence we consider

$$
\begin{aligned}
& 0=x^{*} Q x=\underbrace{x^{*}\left(\beta \mathcal{L}_{k} C \mathcal{L}_{k}\right) x}_{\triangleq a_{0}} \\
& +\underbrace{s x^{*}\left(\gamma I_{n}+\frac{1}{\alpha} \mathcal{L}_{k}+\beta \mathcal{L}_{k} C D\right) x}_{\triangleq a_{1}} \\
& +s^{2} \underbrace{x^{*}\left(I_{n}+\frac{1}{\alpha} D+\beta \mathcal{L}_{k} C M^{-1}\right)}_{\triangleq a_{2}} x+s^{3} \underbrace{x^{*}\left(\frac{1}{\alpha} M^{-1}\right)}_{\triangleq a_{3}} x .
\end{aligned}
$$


We distinguish between two cases. $x^{*} \mathcal{L}_{k} C \mathcal{L}_{k} x=0$, and $x^{*} \mathcal{L}_{k} C \mathcal{L}_{k} x \neq 0$. First consider the case when $x^{*} \mathcal{L}_{k} C M \mathcal{L}_{k} x=0$. Equation (16) may now be written

$$
s a_{1}+s^{2} a_{2}+s^{3} a_{3}=s\left(a_{1}+s a_{2}+s^{2} a_{3}\right)=0 .
$$

The above equation has one solution $s=0$, and two solutions $s \in \mathbb{C}^{-}$if and only if $a_{i}>0, i=1,2,3$ by the RouthHurwitz stability criterion. We now proceed with the case when $x^{*} \mathcal{L}_{k} C \mathcal{L}_{k} x \neq 0$. Since $x^{*} \mathcal{L}_{k} C \mathcal{L}_{k} x \geq 0$, we must have that $x^{*} \mathcal{L}_{k} C \mathcal{L}_{k} x>0$. The Routh-Hurwitz stability criterion is $a_{i}>0$ for $i=0,1,2,3$, and $a_{0} a_{3}<a_{1} a_{2}$. Clearly $a_{0}>0$ and $a_{3}>0$. Consider:

$$
a_{1}=\gamma+x^{*} \frac{1}{\alpha} \mathcal{L}_{k} x+x^{*} \beta \mathcal{L}_{k} C D x .
$$

Clearly $x^{*} \frac{1}{\alpha} \mathcal{L}_{k} x \geq 0$, and since $x^{*} \beta \mathcal{L}_{k} C D x=$ $\frac{1}{2} \beta x^{*}\left(\mathcal{L}_{k} C D+D C \mathcal{L}_{k}\right) x$, we conclude that $a_{1}>0$ if

$$
\beta \lambda_{\min }\left(\frac{1}{2}\left(L_{k} C D+D C L_{k}\right)\right)+\gamma>0 .
$$

By similar arguments it can be shown that $a_{2}>0$ if

$$
\beta \lambda_{\min }\left(\frac{1}{2}\left(L_{k} C M^{-1}+M^{-1} C L_{k}\right)\right)+1+\frac{D}{\alpha}>0 .
$$

Finally the condition $a_{0} a_{3}<a_{1} a_{2}$ can be guaranteed by bounding the left hand side from above, and the right hand side from below. The following bounds are easily verified:

$$
\begin{aligned}
& a_{0} \leq \beta \lambda_{\max }\left(\mathcal{L}_{k} C \mathcal{L}_{k}\right) \\
& a_{3} \leq \frac{\underline{m}}{\alpha} \\
& a_{1} \geq \beta \lambda_{\min }\left(\frac{1}{2}\left(L_{k} C D+D C L_{k}\right)\right)+\gamma \\
& a_{2} \geq \beta \lambda_{\min }\left(\frac{1}{2}\left(L_{k} C M^{-1}+M^{-1} C L_{k}\right)\right)+1+\frac{D}{\alpha} .
\end{aligned}
$$

By substituting $a_{i}, i=0,1,2,3$ with the above bounds we obtain (13).

\section{Centralized CONTROL}

\section{A. Proposed control protocol}

To compare performance with the distributed controller proposed in section IV-A, we propose the following decentralized dynamic controller:

$$
\begin{aligned}
& u_{i}=\alpha\left(\hat{\omega}_{i}-\omega_{i}\right) \\
& \dot{\hat{\omega}}_{i}=\beta\left(u^{*}-\alpha\left(\hat{\omega}_{i}-\omega_{i}\right)\right)+\gamma\left(\omega^{\text {ref }}-\omega_{i}\right),
\end{aligned}
$$

where $\alpha, \beta, \gamma \in \mathbb{R}^{+}$, and $u^{*}$ is given by solving the following centralized optimization program

$$
\begin{aligned}
& {\left[u^{*}, \delta^{*}\right]=\underset{u, \delta}{\operatorname{argmin}} \sum_{i \in \mathcal{V}} \frac{1}{2} C_{i} u_{i}^{2}} \\
& \text { s.t. } \quad \mathcal{L}_{k} \delta-u=P^{m}-\omega^{\mathrm{ref}} D 1_{n \times 1} .
\end{aligned}
$$

Note that solving the above optimization program requires global knowledge about the power load $P^{m}$, the power generation $\operatorname{costs} C$, as well as an exact model of the power system. We will show that the controller (17) satisfies conditions 1 and 2.

\section{B. Sufficient stability criteria based on eigenvalues}

In this section we study the stability of (3) with the control given by (17). We first give sufficient conditions for the stability of the proposed control protocol based on linear system theory.

Theorem 3: The power system (3) with control input (17) satisfies Conditions 1 and 2 for any initial condition $(\delta(0), \omega(0))$ if the matrix

$$
A \triangleq\left[\begin{array}{ccc}
-\alpha \beta I_{n} & 0_{n \times n} & (\alpha \beta-\gamma) I_{n} \\
0_{n \times n} & 0_{n \times n} & I_{n} \\
\alpha M & -M \mathcal{L}_{k} & -M\left(D+\alpha I_{n}\right)
\end{array}\right]
$$

has exactly one eigenvalue equal to 0 and all other eigenvalues in the left half complex plane.

Proof: The proof is analogous with the proof of Theorem 1 and is omitted.

While Theorem 3 provides a straightforward condition weather a given set of parameters result in a stable system, it does not give any implication on how to stabilize an unstable system. The following theorem gives a sufficient conditions for when $A$ has all eigenvalues except one in the open left half complex plane, analogue to the conditions in theorem 2.

Theorem 4: $A$ has exactly one zero eigenvalue, and all other eigenvalues in the left half complex plane if the following condition is satisfied

$$
\beta \bar{m} \lambda_{\max }\left(\mathcal{L}_{k}\right)<(\gamma+\beta \underline{D})(\alpha \underline{D}+\alpha \beta \underline{m}) .
$$

where $\underline{m}=\min _{i} m_{i}, \underline{m}=\min _{i} m_{i}$ and $\underline{D}=\min _{i} D_{i}$.

Remark 2: Given the power system parameters and the controller gains $\alpha$ and $\gamma$, there always exists $\beta>0$, such that the controller (17) stabilizes the power system.

Proof: The characteristic polynomial of $A$ is given by:

$$
\begin{aligned}
& 0=\left|\begin{array}{ccc}
(-\alpha \beta-s) I_{n} & 0_{n \times n} & (\alpha \beta-\gamma) I_{n} \\
0_{n \times n} & -s I_{n} & I_{n} \\
\alpha M & -M \mathcal{L}_{k} & -M D-\alpha M-s I_{n}
\end{array}\right| \\
& =\left|\begin{array}{ccc}
(-\alpha \beta-s) I_{n} & 0_{n \times n} & (-\gamma-s) I_{n} \\
0_{n \times n} & -s I_{n} & I_{n} \\
\alpha M & -M \mathcal{L}_{k} & -M D-s I_{n}
\end{array}\right| \\
& =\frac{1}{s^{2}}\left|\begin{array}{ccc}
(-\alpha \beta-s) I_{n} & 0_{n \times n} & \left(-\gamma s-s^{2}\right) I_{n} \\
0_{n \times n} & -s I_{n} & 0_{n \times n} \\
\alpha M & -M \mathcal{L}_{k} & -s M D-s^{2} I_{n}-M \mathcal{L}_{k}
\end{array}\right| \\
& =\left|\begin{array}{ccc}
(-\alpha \beta-s) I_{n} & 0_{n \times n} & \left(-\gamma s-s^{2}\right) I_{n} \\
0_{n \times n} & -I_{n} & 0_{n \times n} \\
\alpha M & 0_{n \times n} & -s M D-s^{2} I_{n}-M \mathcal{L}_{k}
\end{array}\right| \\
& =\left|\begin{array}{cc}
(-\alpha \beta-s) I_{n} & \left(-\gamma s-s^{2}\right) I_{n} \\
\alpha M & -s M D-s^{2} I_{n}-M \mathcal{L}_{k}
\end{array}\right| \\
& =\alpha \operatorname{det} M \operatorname{det}\left((\alpha \beta+s)\left(M \mathcal{L}_{k} s M D+s^{2} I_{n}\right)\right. \\
& \left.+\left(\gamma s+s^{2} I_{n}\right)\right) \text {. }
\end{aligned}
$$

Clearly the above characteristic equation has a solution only if

$$
x^{*}\left((\alpha \beta+s)\left(M \mathcal{L}_{k} s M D+s^{2} I_{n}\right)+\left(\gamma s+s^{2} I_{n}\right)\right) x=0 .
$$


has a solution. Hence if (19) has all its solutions in $\mathbb{C}^{-}$for all $\|x\|=1$, then (18) has all its solutions in $\mathbb{C}^{-}$. This condition thus becomes that the equation

$$
\begin{aligned}
& \underbrace{x^{*} \beta \mathcal{L}_{k} x}_{a_{0}}+\underbrace{s x^{*}\left(\gamma I_{n}+\frac{1}{\alpha} \mathcal{L}_{k}+\beta D\right)}_{a_{1}} x \\
& +s^{2} \underbrace{x^{*}\left(I_{n}+\frac{1}{\alpha}\right)}_{a_{2}} x+s^{3} \underbrace{\frac{1}{\alpha} x^{*} M^{-1} x}_{a_{3}}=0,
\end{aligned}
$$

has all its solutions in $\mathbb{C}^{-}$. We distinguish between the two cases: $x^{*} \mathcal{L}_{k} x=0$ and $x^{*} \mathcal{L}_{k} x \neq 0$. Starting with the former case, equation (16) may be written as

$$
s a_{1}+s^{2} a_{2}+s^{3} a_{3}=s\left(a_{1}+s a_{2}+s^{2} a_{3}\right)=0
$$

If $a_{i}>0$ for $i=1,2,3$, the above equation has one solution $s=0$, and two solutions $s \in \mathbb{C}^{-}$if and only if $a_{i}>0$, $i=1,2,3$ by the Routh-Hurwitz stability criterion. We now proceed with the case when $x^{*} \mathcal{L}_{k} x \neq 0$. Since $x^{*} \mathcal{L}_{k} x \geq 0$, we conclude that $x^{*} \mathcal{L}_{k} x>0$. The Routh-Hurwitz stability criterion is $a_{i}>0$ for $i=0,1,2,3$, and $a_{0} a_{3}<a_{1} a_{2}$. Clearly $a_{i}>0$ for $i=0,1,2,3$, and the latter condition becomes

$$
\begin{aligned}
& x^{*} \beta \mathcal{L}_{k} x \frac{1}{\alpha} x^{*} M^{-1} x \\
& <x\left(\gamma I_{n}+\frac{1}{\alpha} \mathcal{L}_{k}+\beta D\right) x x^{*}\left(I_{n}+\frac{1}{\alpha}\right) x .
\end{aligned}
$$

A sufficient condition for the above equation to hold is obtained by upper bounding the left hand side and lower bounding the right hand side, which yields

$$
\beta \bar{m} \lambda_{\max }\left(\mathcal{L}_{k}\right)<(\gamma+\beta \underline{D})(\alpha \underline{D}+\alpha \beta \underline{m}) .
$$

\section{Simulations}

The centralized and distributed frequency control algorithms were tested on the IEEE 30 bus test system [13]. The line admittances were extracted from [13] and the voltages were assumed to be $132 \mathrm{kV}$ for all buses. The values of $M$ and $D$ were assumed to be given by $m_{i}=10^{5} \mathrm{~kg} \mathrm{~m}^{2}$ and $d_{i}=1 s^{-1} \forall i \in \mathcal{V}$. The dynamics of the power system were modeled by the nonlinear swing equation (1). The power system is initially in an operational equilibrium, until the power load is increased by a step of $20 \mathrm{~kW}$ in the buses 2,3 and 7 . This will immediately result in decreased frequencies at the load buses. The frequency controllers at the buses will then control the frequencies towards the desired frequency of $\omega^{\text {ref }}=50 \mathrm{~Hz}$. When simulating the centralized controller the parameters were set to $\alpha=5 \cdot 10^{4}, \beta=5 \cdot 10^{-11}$, $\gamma=0.02$, while when simulating the distributed control architecture the parameters were $\alpha=5 \cdot 10^{4}, \beta=5 \cdot 10^{-6}$, $\gamma=0.2$. The choice of parameters was verified to stabilize the power system using Theorems 2 and 4, respectively. Note that the difference in parameters between the two controller architectures is due to the line susceptances $k_{i j}$ are being integrated in the distributed controller (7), as opposed to in the centralized controller (17).
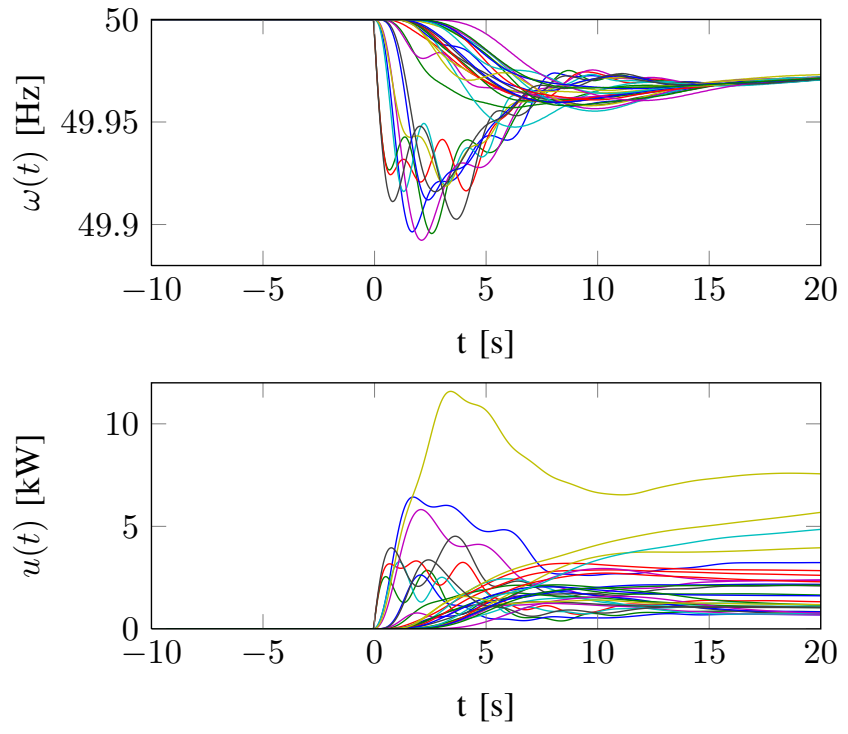

Figure 1. The upper figure shows the transient bus frequencies over $10 \mathrm{~s}$, while the lower figure shows the control inputs, when using the distributed controller.
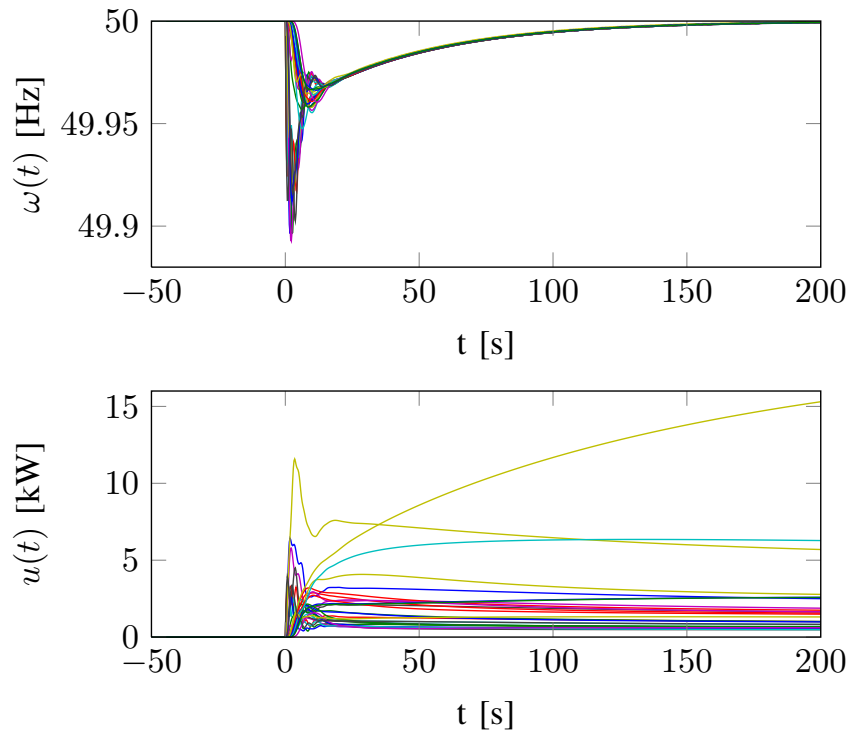

Figure 2. The upper figure shows the bus frequencies over $200 \mathrm{~s}$, while the lower figure shows the control inputs, when using the distributed controller.

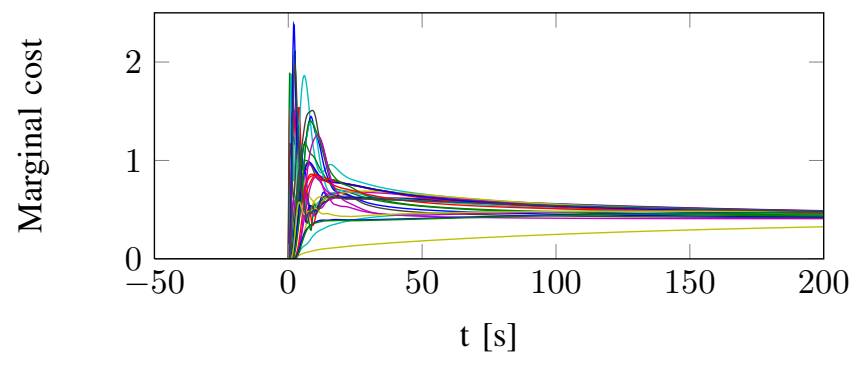

Figure 3. The figure shows the costs of the power generation of the buses, when using the distributed controller. 

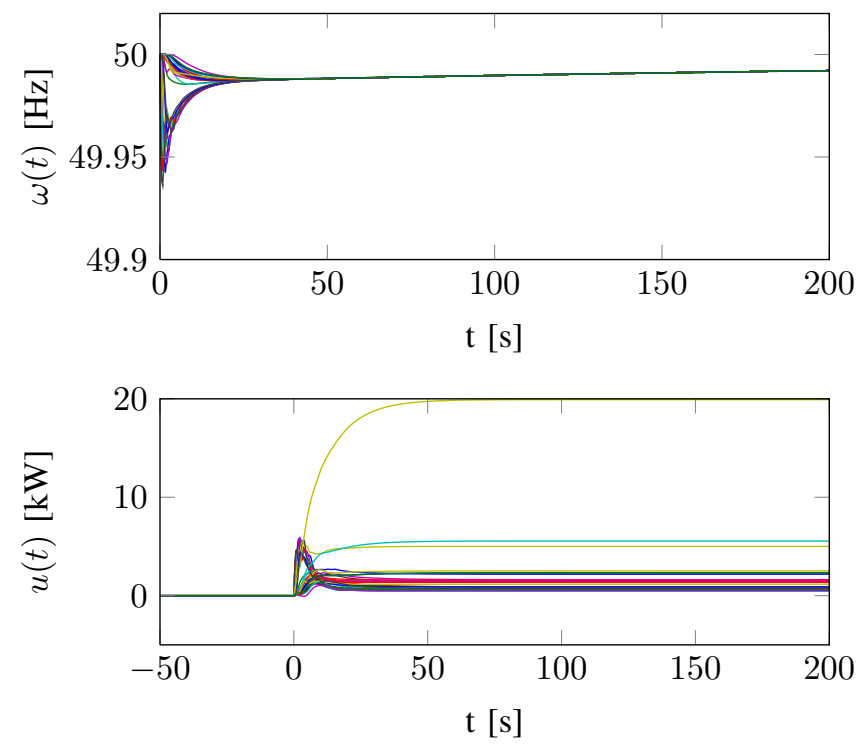

Figure 4. The upper figure shows the bus frequencies over $100 \mathrm{~s}$, while the lower figure shows the control inputs, when using the centralized controller.

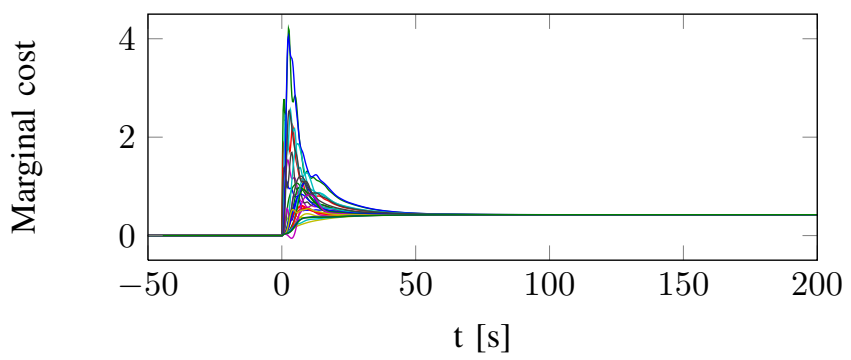

Figure 5. The figure shows the costs of the power generation of the buses, when using the centralized controller.

As seen in Figure 2, the distributed controller quickly regulates the bus frequencies towards a common frequency, which is subsequently regulated towards the reference frequency. The generation costs, as seen in figure 3 are also slowly regulated towards the optimal costs. The centralized controller on the other hand regulates the both the bus frequencies and the generation costs towards their optimal values several times faster than the corresponding distributed controller, compare figure 3 and 5. The centralized controller is able to stabilize the system faster since the optimal generation profile is known a priori, whereas it is unknown a priori for the distributed controller.

\section{DISCUSSION AND CONCLUSIONS}

In this paper we have considered a distributed controller with PI structure for electrical power systems. We have shown that the proposed controller regulates the bus frequencies of the power system towards a common reference frequency (e.g., $50 \mathrm{HZ}$ ), while the power generation profile at the equilibrium minimizes a quadratic cost function. We have provided sufficient conditions for the control parameters, under which the controller stabilizes the system. Furthermore we have derived non-tight sufficient stability conditions which prove to be more easily verified. For performance comparison, we have also considered a centralized controller fulfilling the same control objectives as the distributed controller. Sufficient, as well as non-tight sufficient stability criteria were derived for this controller.

Simulations on the IEEE 30 bus test power network show that both controllers have acceptable performance. However, the centralized controller is considerably faster than the distributed controller. The centralized controller however requires global knowledge about the power system parameters, as well the load and generation profile of the whole power system. Whenever this information is not globally available, distributed control is the only viable option. Future work will address optimizing the transient response of the distributed controller and exploring tighter stability criteria.

\section{REFERENCES}

[1] N. Jaleeli, L.S. VanSlyck, D.N. Ewart, L.H. Fink, and A.G. Hoffmann. Understanding automatic generation control. Power Systems, IEEE Transactions on, 7(3):1106 -1122, aug 1992.

[2] Ibraheem, P. Kumar, and D.P. Kothari. Recent philosophies of automatic generation control strategies in power systems. Power Systems, IEEE Transactions on, 20(1):346 - 357, feb. 2005.

[3] F. Liu, Y.H. Song, J. Ma, S. Mei, and Q. Lu. Optimal load-frequency control in restructured power systems. Generation, Transmission and Distribution, IEE Proceedings-, 150(1):87 - 95, jan. 2003.

[4] J. Machowski, J.W. Bialek, and J.R. Bumby. Power System Dynamics: Stability and Control. Wiley, 2008.

[5] H. Bevrani. Robust power system frequency control. Springer, 2009.

[6] A.N. Venkat, I.A. Hiskens, J.B. Rawlings, and S.J. Wright. Distributed mpc strategies with application to power system automatic generation control. Control Systems Technology, IEEE Transactions on, 16(6):1192-1206, 2008.

[7] M. Morari and E. Zafiriou. Robust Process Control. Prentice Hall, Englewood Cliffs, 1989.

[8] N. Senroy, G.T. Heydt, and V. Vittal. Decision tree assisted controlled islanding. Power Systems, IEEE Transactions on, 21(4):1790 -1797, nov. 2006.

[9] Bo Yang, V. Vittal, and G.T Heydt. Slow-coherency-based controlled islanding: A demonstration of the approach on the august 14, 2003 blackout scenario. Power Systems, IEEE Transactions on, 21(4):18401847, nov. 2006.

[10] M. Andreasson, H Sandberg, D. V. Dimarogonas, and K. H. Johansson. Distributed integral action: Stability analysis and frequency control of power systems. In IEEE Conference on Decision and Control, dec. 2012.

[11] W. Karush. Minima of functions of several variables with inequalities as side conditions. 1939.

[12] J. R. Silvester. Determinants of block matrices. The Mathematical Gazette, 84(501):pp. 460-467, 2000.

[13] Power systems test case archive-30 bus power flow test case. available in: http://www.ee.washington.edu/research/pstca/pf30/pgtca30bus.htm. 